\title{
ON NONNEGATIVE REALIZATION OF PARTITIONED SPECTRA*
}

\author{
RICARDO L. SOTO ${ }^{\dagger}$, OSCAR ROJO ${ }^{\dagger}$, AND CRISTINA B. MANZANEDA ${ }^{\dagger}$
}

\begin{abstract}
We consider partitioned lists of real numbers $\Lambda=\left\{\lambda_{1}, \lambda_{2}, \ldots, \lambda_{n}\right\}$, and give efficient and constructive sufficient conditions for the existence of nonnegative and symmetric nonnegative matrices with spectrum $\Lambda$. Our results extend the ones given in [R.L. Soto and O. Rojo. Applications of a Brauer theorem in the nonnegative inverse eigenvalue problem. Linear Algebra Appl., 416:844856, 2006.] and [R.L. Soto, O. Rojo, J. Moro, and A. Borobia. Symmetric nonnegative realization of spectra. Electron. J. Linear Algebra, 16:1-18, 2007.] for the real and symmetric nonnegative inverse eigenvalue problem. We also consider the complex case and show how to construct an $r \times r$ nonnegative matrix with prescribed complex eigenvalues and diagonal entries.
\end{abstract}

Key words. Nonnegative inverse eigenvalue problem.

AMS subject classifications. 15A18.

1. Introduction. The nonnegative inverse eigenvalue problem (NIEP) is the problem of characterizing all possible spectra of entrywise nonnegative matrices. This problem remains unsolved. A complete solution is known only for $n \leq 4$ [9, 11, 22]. Sufficient conditions for the existence of a nonnegative matrix with prescribed complex spectrum have been obtained in $[2,13,14]$, and recently in [20]. Necessary conditions have been found in $[1,7,9]$. A list $\Lambda=\left\{\lambda_{1}, \lambda_{2}, \ldots, \lambda_{n}\right\}$ of complex numbers is said to be realizable if $\Lambda$ is the spectrum of an entrywise nonnegative matrix $A$. In this case $A$ is said to be a realizing matrix. If $\Lambda$ is a list of real numbers, then we have the real nonnegative inverse eigenvalue problem (RNIEP) (see $[3,10,15]$ and references therein). In [10] the authors construct a map of the sufficient conditions for the RNIEP and establish inclusion relations or independency relations between different sufficient conditions. If the realizing matrix is required to be symmetric, then we have the symmetric nonnegative inverse eigenvalue problem (SNIEP) [5, 8, 13, 17, 19, 21].

It is well-known that if $\Lambda$ is the spectrum of an $n \times n$ nonnegative matrix $A$, then $\rho(A)=\max _{1 \leq i \leq n}\left|\lambda_{i}\right|$ is an eigenvalue of $A$. This eigenvalue is called the Perron eigenvalue of $A$ and we shall always assume, in this paper, that $\rho(A)=\lambda_{1}$. A matrix $A=\left[a_{i j}\right]$ is said to have constant row sums if all its rows sum up to the same constant,

* Received by the editors on November 24, 2010. Accepted for publication on May 8, 2011. Handling Editor: Michael Neumann.

${ }^{\dagger}$ Departamento de Matemáticas, Universidad Católica del Norte, Casilla 1280, Antofagasta, Chile (rsoto@ucn.cl, orojo@ucn.cl, crismanzaneda@gmail.com). Supported by Fondecyt 1085125, Chile. This work was finished while the two first authors were visiting Universidade de Aveiro, Portugal. 
say $\alpha$, i.e.,

$$
\sum_{j=1}^{n} a_{i j}=\alpha, \quad i=1, \ldots, n .
$$

The set of all matrices with constant row sums equal to $\alpha$ will be denoted as $\mathcal{C} \mathcal{S}_{\alpha}$. It is clear that any matrix in $\mathcal{C S}_{\alpha}$ has eigenvector $\mathbf{e}=(1,1, \ldots, 1)^{T}$ corresponding to the eigenvalue $\alpha$. We shall denote by $\mathbf{e}_{k}$ the $n$-dimensional vector with one in the $k$ th position and zeros elsewhere.

It is clear that if $\Lambda=\left\{\lambda_{1}, \lambda_{2}, \ldots, \lambda_{n}\right\}$ can be partitioned as $\Lambda=\Lambda_{1} \cup \cdots \cup$ $\Lambda_{r}$ in such a way that each $\Lambda_{i}$ is realizable by a nonnegative matrix $A_{i}$, then $\Lambda$ is realizable by the nonnegative block diagonal matrix $A=\oplus_{i} A_{i}$. In $[2,16]$ the authors give conditions under which $\Lambda$ is realizable even if some of the $\Lambda_{i}$ 's are not realizable, provided there are other sublists $\Lambda_{j}$ which are realizable and, in a certain way, compensate the nonrealizability of the former ones. This is done by employing a very useful result, due to Brauer [4], together with the properties of real matrices with constant row sums. The Brauer result, which has played a relevant role in the study of the NIEP, shows how to modify one single eigenvalue of a matrix via a rank-1 perturbation, without changing any of the remaining eigenvales. The real matrices with constant row sums are important because it is known that the problem of finding a nonnegative matrix with spectrum $\Lambda=\left\{\lambda_{1}, \ldots, \lambda_{n}\right\}$ is equivalent to the problem of finding a nonnegative matrix with constant row sums $\lambda_{1}$ and spectrum $\Lambda$.

In [12], Perfect presents an extension of the Brauer result in [4], which shows how to modify $r$ eigenvalues of a matrix of order $n, r \leq n$, via a $r a n k-r$ perturbation, without changing any of the $n-r$ remaining eigenvalues. By using this extension Perfect gives, in [12], a realizability criterion for partitioned lists of real numbers. She points out that the Brauer extension and its proof are due to Professor R. Rado. In [18], the authors extend the Perfect's result by increasing the number of ways in which the given list can be partitioned, obtaining then a new realizability criterion, which so far appeared to be one of the most general sufficient condition for the RNIEP. In this paper, we shall extend this sufficient condition. In [19], the authors prove a symmetric version of the Rado's result, and from it, they obtain a new symmetric realizability criterion for partitioned lists, which will also be extended in this paper. Recently, in [20], the authors have derived an efficient and constructive sufficient condition for the existence of a nonnegative matrix with a prescribed complex spectrum. This condition can be viewed as a complex version of the result in [18].

We shall need the following four results. The first two are the Brauer extension and its symmetric version. The last two, which will be extended in this paper, give the constructive sufficient conditions for the real and symmetric cases mentioned above.

Theorem 1.1 (Rado, [12]). Let $A$ be an $n \times n$ matrix (complex or real) with 
eigenvalues $\lambda_{1}, \lambda_{2}, \ldots, \lambda_{n}$ and corresponding eigenvectors $\mathbf{x}_{i}$ satisfying $A \mathbf{x}_{i}=\lambda_{i} \mathbf{x}_{i}$. Let $X=\left[\mathbf{x}_{1}\left|\mathbf{x}_{2}\right| \cdots \mid \mathbf{x}_{r}\right]$ be such that $\operatorname{rank}(X)=r$ with $r \leq n$. Let $C$ be an $r \times n$ matrix (complex or real). Then $A+X C$ has eigenvalues $\mu_{1}, \ldots, \mu_{r}, \lambda_{r+1}, \ldots, \lambda_{n}$, where $\mu_{1}, \ldots, \mu_{r}$ are eigenvalues of the matrix $\Omega+C X$ with $\Omega=\operatorname{diag}\left(\lambda_{1}, \ldots, \lambda_{r}\right)$.

ThEOREM 1.2 ([19]). Let $A$ be an $n \times n$ symmetric matrix with eigenvalues $\lambda_{1}, \ldots, \lambda_{n}$, and for some $r \leq n$, let $\left\{\mathbf{x}_{1}, \mathbf{x}_{2}, \ldots, \mathbf{x}_{r}\right\}$ be an orthonormal set of eigenvectors of $A$ satisfying $A \mathbf{x}_{i}=\lambda_{i} \mathbf{x}_{i}$. Let $X$ be the $n \times r$ matrix with the ith column equal to $\mathbf{x}_{i}, \Omega=\operatorname{diag}\left(\lambda_{1}, \ldots, \lambda_{r}\right)$, and $C$ be any $r \times r$ symmetric matrix. Then the symmetric matrix $A+X C X^{T}$ has eigenvalues $\mu_{1}, \mu_{2}, \ldots, \mu_{r}, \lambda_{r+1}, \ldots, \lambda_{n}$, where $\mu_{1}, \mu_{2}, \ldots, \mu_{r}$ are the eigenvalues of the matrix $\Omega+C$.

THEOREM 1.3 ([18]). Let $\Lambda=\left\{\lambda_{1}, \lambda_{2}, \ldots, \lambda_{n}\right\}$ be a list of real numbers with $\lambda_{1} \geq \lambda_{2} \geq \cdots \geq \lambda_{n}$ and, for some $r \leq n$, let $\omega_{1}, \ldots, \omega_{r}$ be real numbers satisfying $0 \leq \omega_{k} \leq \lambda_{1}, k=1, \ldots, r$. Suppose that the following two conditions hold:

i) There exists a partition $\Lambda=\Lambda_{1} \cup \cdots \cup \Lambda_{r}$, with

$$
\begin{aligned}
& \Lambda_{k}=\left\{\lambda_{k 1}, \lambda_{k 2}, \ldots, \lambda_{k p_{k}}\right\} ; \quad \lambda_{k 1} \geq 0, \quad \lambda_{11}=\lambda_{1} \\
& \lambda_{k 1} \geq \lambda_{k 2} \geq \cdots \geq \lambda_{k p_{k}}, \quad k=1,2, \ldots, r,
\end{aligned}
$$

such that for each $k=1, \ldots, r, \Gamma_{k}=\left\{\omega_{k}, \lambda_{k 2}, \ldots, \lambda_{k p_{k}}\right\}$ is a realizable list;

ii) there exists an $r \times r$ nonnegative matrix $B \in \mathcal{C S}_{\lambda_{1}}$ with eigenvalues $\lambda_{11}, \lambda_{21}$, $\ldots, \lambda_{r 1}$ and diagonal entries $\omega_{1}, \omega_{2}, \ldots, \omega_{r}$.

Then $\Lambda$ is realizable.

TheOREM 1.4 ([19]). Let $\Lambda=\left\{\lambda_{1}, \lambda_{2}, \ldots, \lambda_{n}\right\}$ be a list of real numbers with $\lambda_{1} \geq \lambda_{2} \geq \cdots \geq \lambda_{n}$ and, for some $r \leq n$, let $\omega_{1}, \ldots, \omega_{r}$ be real numbers satisfying $0 \leq \omega_{k} \leq \lambda_{1}, k=1, \ldots, r$. Suppose that the following two conditions hold:

i) There exists a partition $\Lambda=\Lambda_{1} \cup \cdots \cup \Lambda_{r}$, with

$$
\begin{aligned}
\Lambda_{k} & =\left\{\lambda_{k 1}, \lambda_{k 2}, \ldots, \lambda_{k p_{k}}\right\} ; \quad \lambda_{k 1} \geq 0, \quad \lambda_{11}=\lambda_{1} \\
\lambda_{k 1} & \geq \lambda_{k 2} \geq \cdots \geq \lambda_{k p_{k}}, \quad k=1,2, \ldots, r,
\end{aligned}
$$

such that for each $k=1, \ldots, r$, the list $\Gamma_{k}=\left\{\omega_{k}, \lambda_{k 2}, \ldots, \lambda_{k p_{k}}\right\}$ is realizable by a symmetric nonnegative matrix of order $p_{k}$;

ii) there exists an $r \times r$ symmetric nonnegative matrix $B$ with eigenvalues $\lambda_{11}$, $\lambda_{21}, \ldots, \lambda_{r 1}$ and diagonal entries $\omega_{1}, \omega_{2}, \ldots, \omega_{r}$.

Then $\Lambda$ is realizable by an $n \times n$ symmetric nonnegative matrix.

This paper is organized as follows: Section 2 is devoted to constructing nonneg- 
ative matrices with prescribed eigenvalues and diagonal entries. In Section 3 we give a new efficient and constructive sufficient condition for the real case, extending the result in Theorem 1.3. In Section 4, we extend the symmetric realizability criterion given in Theorem 1.4. We also consider, in Section 5, the complex case.

2. Nonnegative matrices with prescribed eigenvalues and diagonal entries. To make use of Theorem 1.3 and its extension in Section 3, we need to know conditions under which there exists an $r \times r$ nonnegative matrix $B \in \mathcal{C S}_{\lambda_{1}}$ with eigenvalues $\lambda_{1}, \lambda_{2}, \ldots, \lambda_{r}$ and diagonal entries $\omega_{1}, \omega_{2}, \ldots, \omega_{r}$. For $r=2$, there exists a nonnegative matrix with eigenvalues $\lambda_{1}, \lambda_{2}$ and diagonal entries $\omega_{1}, \omega_{2}$, if and only if $0 \leq \omega_{k} \leq \lambda_{1}, k=1,2$, and $\omega_{1}+\omega_{2}=\lambda_{1}+\lambda_{2}$. Of course, in this case, the eigenvalues must be real. For $r=3$ we have, in the complex case, the following result given in [20], which slightly extends a result for the real case due to Perfect [12].

TheOREM 2.1. The numbers $\lambda_{1}, \lambda_{2}, \lambda_{3}, \lambda_{1} \geq\left|\lambda_{i}\right|, i=2,3$, (with $\lambda_{2} \geq \lambda_{3}$ for $\lambda_{2}, \lambda_{3}$ real), and $\omega_{1}, \omega_{2}, \omega_{3}$ are, respectively, the eigenvalues and the diagonal entries of a $3 \times 3$ nonnegative matrix $B \in \mathcal{C S}_{\lambda_{1}}$ if and only if

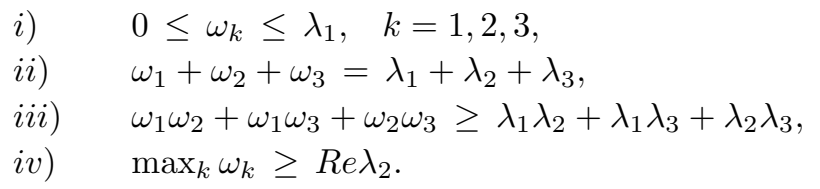

For $r \geq 4$, only sufficient conditions are known. In [20], lists of $r$ numbers of the form $\Lambda=\left\{\lambda_{1}, \ldots, \lambda_{r-2}, a+b i, a-b i\right\}$, where $\lambda_{1}, \ldots, \lambda_{r-2}$ are real, are considered, and a sufficient condition for the existence of a nonnegative matrix with such spectrum and diagonal entries $\omega_{1}, \ldots, \omega_{r}$ is given. Here we have:

THEOREM 2.2. Let the lists $\Lambda=\left\{\lambda_{1}, a+b i, a-b i, c+d i, c-d i\right\}$ and $\left\{\omega_{1}, \omega_{2}, \ldots, \omega_{5}\right\}$ be given. Let $\mu=\omega_{1}+\omega_{2}+\omega_{3}-2 a$. If the numbers

i) $\mu, c+d i, c-d i$ and $\omega_{1}, \omega_{2}, \omega_{3}$

and

$$
\text { ii) } \lambda_{1}, a+b i, a-b i \quad \text { and } \quad \mu, \omega_{4}, \omega_{5}
$$

satisfy conditions of Theorem 2.1, that is, if the numbers in $i$ ) and ii) are the eigenvalues and the diagonal entries of $3 \times 3$ nonnegative matrices $A_{1} \in \mathcal{C S}_{\mu}$ and $B \in \mathcal{C} \mathcal{S}_{\lambda_{1}}$, respectively, then there exists a nonnegative matrix $M$ with spectrum $\Lambda$ and diagonal entries $\omega_{1}, \ldots, \omega_{5}$.

Proof. If the lists in $i$ ) and $i i$ ) satisfy Theorem 2.1, then we may construct a 
nonnegative matrix (see [18])

$$
A_{1}=\left[\begin{array}{ccc}
\omega_{1} & 0 & \mu-\omega_{1} \\
\mu-\omega_{2}-p & \omega_{2} & p \\
0 & \mu-\omega_{3} & \omega_{3}
\end{array}\right] \in \mathcal{C S}_{\mu},
$$

where $p=\frac{1}{\mu-\omega_{3}}\left(\omega_{1} \omega_{2}+\omega_{1} \omega_{3}+\omega_{2} \omega_{3}-2 \mu c-c^{2}-d^{2}\right)$, with eigenvalues $\mu, c+d i, c-d i$ and diagonal entries $\omega_{1}, \omega_{2}, \omega_{3}$, and a nonnegative matrix

$$
B=\left[\begin{array}{ccc}
\mu & 0 & \lambda_{1}-\mu \\
\lambda_{1}-\omega_{4}-q & \omega_{4} & q \\
0 & \lambda_{1}-\omega_{5} & \omega_{5}
\end{array}\right] \in \mathcal{C S}_{\lambda_{1}},
$$

where $q=\frac{1}{\lambda_{1}-\omega_{5}}\left(\mu \omega_{4}+\mu \omega_{5}+\omega_{4} \omega_{5}-2 \lambda_{1} a-a^{2}-b^{2}\right)$ with eigenvalues $\lambda_{1}, a+b i, a-b i$ and diagonal entries $\mu, \omega_{4}, \omega_{5}$. Let

$$
A=\left[\begin{array}{lll}
A_{1} & & \\
& \omega_{4} & \\
& & \omega_{5}
\end{array}\right], \quad X=\left[\begin{array}{lll}
1 & 0 & 0 \\
1 & 0 & 0 \\
1 & 0 & 0 \\
0 & 1 & 0 \\
0 & 0 & 1
\end{array}\right]
$$

and let $C$ be the matrix obtained from $B$ as

$$
C=\left[\begin{array}{ccccc}
0 & 0 & 0 & 0 & \lambda_{1}-\mu \\
\lambda_{1}-\omega_{4}-q & 0 & 0 & 0 & q \\
0 & 0 & 0 & \lambda_{1}-\omega_{5} & 0
\end{array}\right] .
$$

Then $M=A+X C$ is nonnegative and from Theorem 1.1 it has spectrum $\Lambda$ and diagonal entries $\omega_{1}, \ldots, \omega_{5}$.

Next we generalize Theorem 2.2.

TheOREM 2.3. Let $\Lambda=\left\{\lambda_{1}, \lambda_{2}, \ldots, \lambda_{n}\right\}$ be a list of complex numbers and let $\Omega=\left\{\omega_{1}, \ldots, \omega_{n}\right\}$ be a list of real numbers satisfying $0 \leq \omega_{k} \leq \lambda_{1}, k=1, \ldots, n$. Suppose that the following two conditions hold:

i) There exist partitions $\Lambda=\Lambda_{1} \cup \cdots \cup \Lambda_{r}$, with $\Lambda_{k}=\left\{\lambda_{k 1}, \lambda_{k 2}, \ldots, \lambda_{k p_{k}}\right\}$, $\lambda_{11}=\lambda_{1}$, and $\Omega=\Omega_{1} \cup \cdots \cup \Omega_{r}$, with $\Omega_{k}=\left\{\omega_{k 1}, \omega_{k 2}, \ldots, \omega_{k p_{k}}\right\}, k=1, \ldots, r$, in such a way that for each $k=1, \ldots, r$, there exists $0 \leq \mu_{k} \leq \lambda_{1}$ and a $p_{k} \times p_{k}$ nonnegative matrix $A_{k} \in \mathcal{C S}_{\mu_{k}}$ with spectrum $\Gamma_{k}=\left\{\mu_{k}, \lambda_{k 2}, \ldots, \lambda_{k p_{k}}\right\}$ and diagonal entries $\omega_{k 1}, \omega_{k 2}, \ldots, \omega_{k p_{k}}$;

ii) there exists an $r \times r$ nonnegative matrix $B$ with eigenvalues $\lambda_{11}, \lambda_{21}, \ldots, \lambda_{r 1}$ (the first elements of lists $\Lambda_{k}$ ) and diagonal entries $\mu_{1}, \mu_{2}, \ldots, \mu_{r}$ (the first elements of lists $\Gamma_{k}$ ). 
Then there exists an $n \times n$ nonnegative matrix with spectrum $\Lambda$ and diagonal entries $\omega_{1}, \omega_{2}, \ldots, \omega_{n}$.

Proof. From $i) A=A_{1} \oplus \cdots \oplus A_{r}$ is an $n \times n$ nonnegative matrix with spectrum $\Gamma_{2} \cup \cdots \cup \Gamma_{r}$ and diagonal entries $\omega_{1}, \ldots, \omega_{n}$. Let $\Omega=\operatorname{diag}\left(\omega_{1}, \ldots, \omega_{r}\right)$ and let $X=$ $\left[\mathbf{x}_{1}|\cdots| \mathbf{x}_{r}\right]$ be an $n \times r$ matrix whose columns are eigenvectors of $A$ corresponding to the eigenvalues $\mu_{k}$. Each $\mathbf{x}_{k}$ can be chosen with $p_{k}$ ones from the position $\sum_{j=1}^{k-1} p_{j}+1$ to the position $\sum_{j=1}^{k} p_{j}$ and zeros elsewhere, with the first summation being zero for $k=1$. Then $X$ is nonnegative and $\operatorname{rank}(X)=r$.

From $i$ ) we construct an $r \times n$ nonnegative matrix $C$ obtained from $B$ in such a way that $B=\Omega+C X$, that is, the first $p_{1}$ columns of $C$ are formed by the first column of $B-\Omega$, followed by $p_{1}-1$ zero columns. The next $p_{2}$ columns of $C$ are formed by the second column of $B-\Omega$, followed by $p_{2}-1$ zero columns, and so on until the last column of $C$, which is formed by the $r$ th column of $B$ (the last column of $B$ ) followed by $p_{r}-1$ zero columns. Thus, from Theorem 1.1, the matrix $M=A+X C$ is nonnegative with spectrum $\Lambda$ and diagonal entries $\omega_{1}, \omega_{2}, \ldots, \omega_{n}$. $\square$

Observe that Theorem 2.3 can also be applied to construct a nonnegative matrix with prescribed real spectrum and diagonal entries.

EXAMPLE 2.4. We want to construct a nonnegative matrix with spectrum $\Lambda=\{11,1+3 i, 1-3 i, 2+i, 2-i, 2+4 i, 2-4 i\}$ and diagonal entries $3,3,3,3,3,3,3$. Take the partition $\Lambda_{1}=\{11,1+3 i, 1-3 i\}, \Lambda_{2}=\{2+4 i, 2+i, 2-i\}, \Lambda_{3}=\{2-4 i\}$, with associated realizable list $\Gamma_{1}=\{7,1+3 i, 1-3 i\}, \Gamma_{2}=\{5,2+i, 2-i\}, \Gamma_{3}=\{3\}$ and $\Omega_{1}=\{3,3,3\}, \Omega_{2}=\{3,3,3\}, \Omega_{3}=\{3\}$. We compute matrices

$$
A_{1}=\left[\begin{array}{ccc}
3 & 0 & 4 \\
\frac{13}{4} & 3 & \frac{3}{4} \\
0 & 4 & 3
\end{array}\right], \quad A_{2}=\left[\begin{array}{ccc}
3 & 1 & 1 \\
2 & 3 & 0 \\
0 & 2 & 3
\end{array}\right], \quad A_{3}=[3]
$$

with spectra $\Gamma_{1}, \Gamma_{2}$ and $\Gamma_{3}$, respectively. We also compute, from Theorem 2.1 a nonnegative matrix $B$ with eigenvalues $11,2+4 i, 2-4 i$ and diagonal entries $7,5,3$. That is

$$
B=\left[\begin{array}{ccc}
7 & 0 & 4 \\
\frac{41}{8} & 5 & \frac{7}{8} \\
0 & 8 & 3
\end{array}\right]
$$

Then

$$
M=\left[\begin{array}{ccc}
A_{1} & & \\
& A_{2} & \\
& & 3
\end{array}\right]+X C, \text { i.e. }
$$


$M=$

$$
=\left[\begin{array}{ccccccc}
3 & 0 & 4 & & & & \\
\frac{13}{4} & 3 & \frac{3}{4} & & & & \\
0 & 4 & 3 & & & & \\
& & & 3 & 1 & 1 & \\
& & & 2 & 3 & 0 & \\
& & & 0 & 2 & 3 & \\
& & & & & & 3
\end{array}\right]+\left[\begin{array}{lll}
1 & 0 & 0 \\
1 & 0 & 0 \\
1 & 0 & 0 \\
0 & 1 & 0 \\
0 & 1 & 0 \\
0 & 1 & 0 \\
0 & 0 & 1
\end{array}\right]\left[\begin{array}{ccccccc}
0 & 0 & 0 & 0 & 0 & 0 & 4 \\
\frac{41}{8} & 0 & 0 & 0 & 0 & 0 & \frac{7}{8} \\
0 & 0 & 0 & 8 & 0 & 0 & 0
\end{array}\right]
$$$$
=\left[\begin{array}{ccccccc}
3 & 0 & 4 & 0 & 0 & 0 & 4 \\
\frac{13}{4} & 3 & \frac{3}{4} & 0 & 0 & 0 & 4 \\
0 & 4 & 3 & 0 & 0 & 0 & 4 \\
\frac{41}{8} & 0 & 0 & 3 & 1 & 1 & \frac{7}{8} \\
\frac{41}{8} & 0 & 0 & 2 & 3 & 0 & \frac{7}{8} \\
\frac{41}{8} & 0 & 0 & 0 & 2 & 3 & \frac{7}{8} \\
0 & 0 & 0 & 8 & 0 & 0 & 3
\end{array}\right]
$$

is the required matrix.

3. The real case. A number of sufficient conditions for a list of real numbers to be the spectrum of an entrywise nonnegative matrix are known. In [10], in order to construct a map of sufficient conditions, the authors compare these conditions and establish inclusion relations or independency relations between them. It is also shown in [10] that Soto-Rojo criterion (Theorem 1.3) contains all realizability criteria for lists of real numbers studied therein. In this section we extend this criterion. The new formulation, although similar to that one of Theorem 1.3, has important differences with it: Again, we deal with partitioned lists $\Lambda=\Lambda_{1} \cup \cdots \cup \Lambda_{r}$, where $\Lambda_{k}=\left\{\lambda_{k 1}, \lambda_{k 2}, \ldots, \lambda_{k p_{k}}\right\}, k=1, \ldots, r$. Now, the first element $\lambda_{k 1}$ need not be nonnegative and the realizable list $\Gamma=\left\{\omega_{k}, \lambda_{k 1}, \ldots, \lambda_{k p_{k}}\right\}$ contains one more element, $\omega_{k}$, which does not replace $\lambda_{k 1}$. Moreover, the number of lists of the partition depend on the number of elements of the firs list $\Lambda_{1}$, which must be realizable as before.

THEOREM 3.1. Let $\Lambda=\left\{\lambda_{1}, \lambda_{2}, \ldots, \lambda_{n}\right\}$ be a list of real numbers with $\lambda_{1} \geq$ $\max _{i}\left|\lambda_{i}\right|(i=2, \ldots, n)$ and $\sum_{i=1}^{n} \lambda_{i} \geq 0$, and let the partition $\Lambda=\Lambda_{1} \cup \cdots \cup \Lambda_{p_{1}+1}$ be such that $\Lambda_{k}=\left\{\lambda_{k 1}, \lambda_{k 2}, \ldots, \lambda_{k p_{k}}\right\}, \lambda_{11}=\lambda_{1}, \lambda_{k 1} \geq \lambda_{k 2} \geq \ldots \geq \lambda_{k p_{k}}$ ( $k=$ $\left.1, \ldots, p_{1}+1\right)$, where $p_{1}$ is the number of elements of the list $\Lambda_{1}$ and some of the lists $\Lambda_{k}$ can be empty. Let $\omega_{2}, \ldots, \omega_{p_{1}+1}$ be real numbers satisfying $0 \leq \omega_{k} \leq \lambda_{1}$, $k=2, \ldots, p_{1}+1$. Suppose that the following two conditions hold:

i) For each $k=2, \ldots, p_{1}+1$, there exits a nonnegative matrix $A_{k} \in \mathcal{C S}_{\omega_{k}}$ with spectrum $\Gamma_{k}=\left\{\omega_{k}, \lambda_{k 1}, \ldots, \lambda_{k p_{k}}\right\}$;

ii) there exists a $p_{1} \times p_{1}$ nonnegative matrix $B \in \mathcal{C S}_{\lambda_{1}}$, with spectrum $\Lambda_{1}$ and 
diagonal entries $\omega_{2}, \ldots, \omega_{p_{1}+1}$.

Then $\Lambda$ is realizable.

Proof. From $i$ ) the matrix $A=A_{2} \oplus A_{3} \oplus \cdots \oplus A_{p_{1}+1}$ is an $n \times n$ nonnegative matrix with spectrum $\Gamma_{2} \cup \cdots \cup \Gamma_{p_{1}+1}$. Let $\Omega=\operatorname{diag}\left(\omega_{2}, \ldots, \omega_{p_{1}+1}\right)$ and let $X=\left[\mathbf{x}_{2}|\cdots| \mathbf{x}_{p_{1}+1}\right]$ be an $n \times p_{1}$ matrix whose columns are eigenvectors of $A$ corresponding to the eigenvalues $\omega_{k}$. Each $\mathbf{x}_{k}, k=2, \ldots, p_{1}+1$, can be chosen with $p_{k}$ ones from the position $\sum_{j=2}^{k-1} p_{j}+1$ to the position $\sum_{j=2}^{k} p_{j}$ and zeros elsewhere, with the first summation being zero for $k=2$. Then $X$ is nonnegative and $\operatorname{rank}(X)=p_{1}$.

From $i i$ ) we construct a $p_{1} \times n$ nonnegative matrix $C$, obtained from $B$ in such a way that $B=\Omega+C X$, that is, the first $p_{2}$ columns of $C$ are formed by the first column of $B-\Omega$, followed by $p_{2}-1$ zero columns. The next $p_{3}$ columns of $C$ are formed by the second column of $B-\Omega$, followed by $p_{3}-1$ zero columns, and so on until the last $p_{p_{1}+1}$ columns of $C$, which are formed by the $p_{1}$ th column of $B$ (the last column of $B$ ) followed by $p_{p_{1}+1}-1$ zero columns. Thus, from Theorem 1.1 , the matrix $M=A+X C$ is nonnegative with spectrum $\Lambda$.

Now we show that Theorem 1.3 implies Theorem 3.1.

Theorem 3.2. If $\Lambda=\left\{\lambda_{1}, \lambda_{2}, \ldots, \lambda_{n}\right\}$ satisfies Soto-Rojo criterion in Theorem 1.3, then $\Lambda$ also satisfies hypotheses of Theorem 3.1.

Proof. Let $\Lambda$ be satisfying Soto-Rojo criterion. Then there exists a partition

$$
\begin{aligned}
\Lambda & =\Lambda_{1} \cup \cdots \cup \Lambda_{r} \quad \text { with } \quad \Lambda_{k}=\left\{\lambda_{k 1}, \lambda_{k 2}, \ldots, \lambda_{k p_{k}}\right\} \\
\lambda_{11} & =\lambda_{1}, \quad \lambda_{k 1} \geq 0, \quad \lambda_{k 1} \geq \lambda_{k 2} \geq \cdots \geq \lambda_{k p_{k}} \quad(k=1,2, \ldots, r),
\end{aligned}
$$

and $\Gamma_{k}=\left\{\omega_{k}, \lambda_{k 2}, \ldots, \lambda_{k p_{k}}\right\}, 0 \leq \omega_{k} \leq \lambda_{1}, k=1, \ldots, r$, being the spectrum of a nonnegative matrix $A_{k} \in \mathcal{C} \mathcal{S}_{\omega_{k}}$. Besides, there exists a nonnegative matrix $B \in \mathcal{C} \mathcal{S}_{\lambda_{1}}$ with eigenvalues $\lambda_{11}, \lambda_{21}, \ldots, \lambda_{r 1}$ and diagonal entries $\omega_{1}, \omega_{2}, \ldots, \omega_{r}$. Then for

$$
\begin{aligned}
& \Lambda_{1}^{\prime}=\left\{\lambda_{11}, \lambda_{21}, \ldots, \lambda_{r 1}\right\}, \Lambda_{k}^{\prime}=\left\{\lambda_{k 2}, \ldots, \lambda_{k p_{k}}\right\}, \quad \text { with } \\
& \Gamma_{k}=\left\{\omega_{k}, \lambda_{k 2}, \ldots, \lambda_{k p_{k}}\right\}, \quad k=1, \ldots, r
\end{aligned}
$$

the hypotheses of Theorem 3.1 are satisfied.

Note that the converse of Theorem 3.2 is not true.

EXAMPLE 3.3. It is known that the list $\Lambda^{\prime}=\{5,4,-3,-3,-3\}$ can not be the spectrum of a nonnegative matrix (it does not satisfy the necessary condition in [7]). Here, we show that $\Lambda=\{5,4,0,-3,-3,-3\}$ is realizable and we compute a realizing 
matrix. Consider the partition

$$
\begin{aligned}
& \Lambda_{1}=\{5,4,0,-3\} ; \Lambda_{2}=\{-3\} ; \Lambda_{3}=\{-3\} \text { with } \\
& \Gamma_{2}=\{3,-3\} ; \Gamma_{3}=\{3,-3\} ; \Gamma_{4}=\Gamma_{5}=\{0\} .
\end{aligned}
$$

Then we look for a nonnegative matrix $B \in \mathcal{C S}_{5}$ with eigenvalues $5,4,0,-3$ and diagonal entries $3,3,0,0$. It is

$$
B=\left[\begin{array}{llll}
3 & 0 & 2 & 0 \\
0 & 3 & 0 & 2 \\
3 & 0 & 0 & 2 \\
0 & 3 & 2 & 0
\end{array}\right]
$$

It is clear that

$$
A_{2}=A_{3}=\left[\begin{array}{ll}
0 & 3 \\
3 & 0
\end{array}\right] \text { realizes } \Gamma_{2}=\Gamma_{3}
$$

Then, from Theorem 1.1, we have that

$$
\begin{aligned}
A & =\left[\begin{array}{llll}
A_{2} & & & \\
& A_{3} & & \\
& & 0 & \\
& & & \\
&
\end{array}\right]+\left[\begin{array}{llll}
1 & 0 & 0 & 0 \\
1 & 0 & 0 & 0 \\
0 & 1 & 0 & 0 \\
0 & 1 & 0 & 0 \\
0 & 0 & 1 & 0 \\
0 & 0 & 0 & 1
\end{array}\right]\left[\begin{array}{llllll}
0 & 0 & 0 & 0 & 2 & 0 \\
0 & 0 & 0 & 0 & 0 & 2 \\
3 & 0 & 0 & 0 & 0 & 2 \\
0 & 0 & 3 & 0 & 2 & 0
\end{array}\right] \\
& =\left[\begin{array}{llllll}
0 & 3 & 0 & 0 & 2 & 0 \\
3 & 0 & 0 & 0 & 2 & 0 \\
0 & 0 & 0 & 3 & 0 & 2 \\
0 & 0 & 3 & 0 & 0 & 2 \\
3 & 0 & 0 & 0 & 0 & 2 \\
0 & 0 & 3 & 0 & 2 & 0
\end{array}\right]
\end{aligned}
$$

has the spectrum $\Lambda$. No partition of $\Lambda$ works for Theorem 1.3.

4. The symmetric case. Several realizability criteria which were obtained for the RNIEP have later been shown to be realizability criteria for the SNIEP as well. Fiedler [5], Radwan [13] and Soto [17] proved, respectively, that criteria of Kellogg, Borobia and Soto (see [10]) obtained for the RNIEP are also symmetric realizability criteria. In [8] Laffey and Šmigoc introduce a symmetric realizability criterion, which combine realizability and diagonal elements of realizing matrices. In [19], the authors give a symmetric version of the Rado's result and, from it, obtain a symmetric realizability criterion, Theorem 1.4 in this paper, which contains criteria of Kellogg, Borobia and Soto. In this Section we extend Theorem 1.4. 
THEOREM 4.1. Let $\Lambda=\left\{\lambda_{1}, \lambda_{2}, \ldots, \lambda_{n}\right\}$ be a list of real numbers with $\lambda_{1} \geq$ $\max _{i}\left|\lambda_{i}\right|(i=2, \ldots, n)$ and $\sum_{i=1}^{n} \lambda_{i} \geq 0$, and let the partition $\Lambda=\Lambda_{1} \cup \cdots \cup \Lambda_{p_{1}+1}$, be such that $\Lambda_{k}=\left\{\lambda_{k 1}, \lambda_{k 2}, \ldots, \lambda_{k p_{k}}\right\}, \lambda_{11}=\lambda_{1}, \lambda_{k 1} \geq \lambda_{k 2} \geq \cdots \geq \lambda_{k p_{k}}$ for $k=1, \ldots, p_{1}+1$, where $\Lambda_{1}$ is symmetrically realizable, $p_{1}$ is the number of elements of the list $\Lambda_{1}$ and some lists $\Lambda_{k}$ can be empty. Let $\omega_{2}, \ldots, \omega_{p_{1}+1}$ be real numbers satisfying $0 \leq \omega_{k} \leq \lambda_{1}, k=2, \ldots, p_{1}+1$. Suppose that the following conditions hold:

i) For each $k=2, \ldots, p_{1}+1$, there exits a symmetric nonnegative matrix $A_{k}$ with spectrum $\Gamma_{k}=\left\{\omega_{k}, \lambda_{k 1}, \ldots, \lambda_{k p_{k}}\right\}$;

ii) there exists a $p_{1} \times p_{1}$ symmetric nonnegative matrix $B$ with spectrum $\Lambda_{1}$ and diagonal entries $\omega_{2}, \ldots, \omega_{p_{1}+1}$.

Then $\Lambda$ is symmetrically realizable.

Proof. From $i$ ) the matrix $A=A_{2} \oplus \cdots \oplus A_{p_{1}+1}$ is symmetric nonnegative with spectrum $\Gamma_{2} \cup \cdots \cup \Gamma_{p_{1}+1}$. Let $\left\{\mathbf{x}_{2}, \ldots, \mathbf{x}_{p_{1}+1}\right\}$ be an orthonormal set of eigenvectors of $A$ associated with $\omega_{2}, \ldots, \omega_{p_{1}+1}$, respectively. Then the $n \times p_{1}$ matrix $X$ with $k$ th column $\mathbf{x}_{k}$ satisfies $A X=X \Omega$ for $\Omega=\operatorname{diag}\left(\omega_{2}, \ldots, \omega_{p_{1}+1}\right)$. Each $\mathbf{x}_{k}$ can be chosen with $p_{k}$ entries, which are the entries of the normalized Perron eigenvector of $A_{k}$, from the position $\sum_{j=2}^{k-1} p_{j}+1$ to the position $\sum_{j=2}^{k} p_{j}$ and zeros elsewhere, with the first summation being zero for $k=2$. Then $X$ is entrywise nonnegative. From ii) $C=B-\Omega$ is symmetric nonnegative and $\Omega+C$ has spectrum $\Lambda_{1}$. Hence, from Theorem 1.2, the symmetric nonnegative matrix $A+X C X^{T}$ has spectrum $\Lambda$.

THEOREM 4.2. If $\Lambda=\left\{\lambda_{1}, \lambda_{2}, \ldots, \lambda_{n}\right\}$ satisfies the symmetric realizability criterion in Theorem 1.4, then $\Lambda$ also satisfies hypotheses of Theorem 4.1.

Proof. Let $\Lambda=\Lambda_{1} \cup \cdots \cup \Lambda_{r}$ with $\Lambda_{k}=\left\{\lambda_{k 1}, \lambda_{k 2}, \ldots, \lambda_{k p_{k}}\right\}$ and $\Gamma_{k}=$ $\left\{\omega_{k}, \lambda_{k 2}, \ldots, \lambda_{k p_{k}}\right\}, 0 \leq \omega_{k} \leq \lambda_{1}, k=1, \ldots, r$, the partition suggested by Teorem 1.4, where the lists $\Gamma_{k}$ are symmetrically realizable and there exists a symmetric nonnegative matrix $B$ with eigenvalues $\lambda_{11}, \lambda_{21}, \ldots, \lambda_{r 1}$ and diagonal entries $\omega_{1}, \omega_{2}, \ldots, \omega_{r}$. Then the partition $\Lambda=\Lambda_{1}^{\prime} \cup \widehat{\Lambda}_{1} \cup \cdots \widehat{\Lambda}_{r}$, where $\Lambda_{1}^{\prime}=\left\{\lambda_{11}, \lambda_{21}, \ldots, \lambda_{r 1}\right\}$, $\widehat{\Lambda}_{k}=\left\{\lambda_{k 2}, \ldots, \lambda_{k p_{k}}\right\}, k=1, \ldots, r$, with $\Gamma_{k}=\left\{\omega_{k}, \lambda_{k 2}, \ldots, \lambda_{k p_{k}}\right\}, k=1, \ldots, r$, satisfies hypotheses of Theorem 4.1.

Note that the Example 4.6 shows that the converse of Theorem 4.2 is not true. In order to apply Theorem 4.1, we need to know under which conditions there exists an $r \times r$ symmetric nonnegative matrix $B$ with eigenvalues $\lambda_{1}, \ldots, \lambda_{r}$ and diagonal entries $\omega_{1}, \ldots, \omega_{r}$. The following necessary and sufficient conditions for a symmetric matrix, not necessarily nonnegative, are due to Horn [6]:

There exists a real symmetric matrix with eigenvalues $\lambda_{1} \geq \lambda_{2} \geq \cdots \geq \lambda_{r}$ and 
diagonal entries $\omega_{1} \geq \omega_{2} \geq \cdots \geq \omega_{r}$ if and only if the vector $\left(\lambda_{1}, \lambda_{2}, \ldots, \lambda_{r}\right)$ majorizes the vector $\left(\omega_{1}, \omega_{2}, \ldots, \omega_{r}\right)$, that is, if and only if

$$
\left.\begin{array}{l}
\sum_{i=1}^{k} \lambda_{i} \geq \sum_{i=1}^{k} \omega_{i}, \quad \text { for } k=1,2, \ldots, r-1 \\
\sum_{i=1}^{r} \lambda_{i}=\sum_{i=1}^{r} \omega_{i}
\end{array}\right\} .
$$

For $r=2$ the conditions (4.1) become $\lambda_{1} \geq \omega_{1}$ and $\lambda_{1}+\lambda_{2}=\omega_{1}+\omega_{2}$, and they are also sufficient for the existence of a $2 \times 2$ symmetric nonnegative matrix with eigenvalues $\lambda_{1} \geq \lambda_{2}$ and diagonal entries $\omega_{1} \geq \omega_{2} \geq 0$. There are also necessary and sufficient conditions for the existence of a $3 \times 3$ symmetric nonnegative matrix with prescribed spectrum and diagonal entries. They are due to Fiedler [5].

LEMma 4.3 ([5]). The following conditions

$$
\left.\begin{array}{rl}
\lambda_{1} & \geq \omega_{1} \\
\lambda_{1}+\lambda_{2} & \geq \omega_{1}+\omega_{2} \\
\lambda_{2}+\lambda_{3} & =\omega_{1}+\omega_{2}+\omega_{3} \\
\lambda_{2} & \leq \omega_{1}
\end{array}\right\}
$$

are necessary and sufficient for the existence of a $3 \times 3$ symmetric nonnegative matrix $B$ with eigenvalues $\lambda_{1} \geq \lambda_{2} \geq \lambda_{3}$ and diagonal entries $\omega_{1} \geq \omega_{2} \geq \omega_{3} \geq 0$.

For $r \geq 4$ we have sufficient conditions due also to Fiedler [5].

Theorem 4.4 ([5]). If $\lambda_{1} \geq \lambda_{2} \geq \cdots \geq \lambda_{r}$ and $\omega_{1} \geq \omega_{2} \geq \cdots \geq \omega_{r}$ satisfy

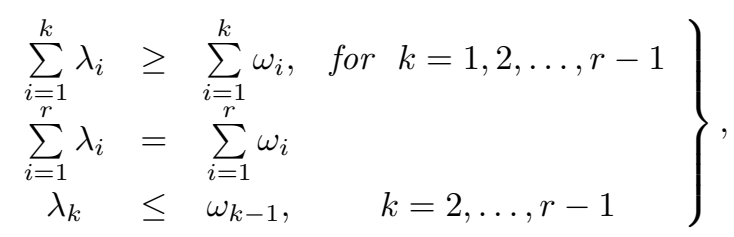

then there exists an $r \times r$ symmetric nonnegative matrix $B$ with eigenvalues $\lambda_{1}, \ldots, \lambda_{r}$ and diagonal entries $\omega_{1}, \ldots, \omega_{r}$.

In [19], using the Fiedler's conditions, the authors show how to construct such a matrix $B$ for $r \geq 3$. Now, from Theorem 4.1, we have the following sufficient condition.

Theorem 4.5. Let $\Lambda=\left\{\lambda_{1}, \ldots, \lambda_{n}\right\}$ and $\Omega=\left\{\omega_{1}, \ldots, \omega_{n}\right\}$ be lists of real numbers, with $\lambda_{1} \geq \max _{i}\left|\lambda_{i}\right|, i=2, \ldots, n ; \sum_{i=1}^{n} \lambda_{i} \geq 0, \quad 0 \leq \omega_{k} \leq \lambda_{1}, k=$ $1, \ldots, n$. Let $\Lambda=\Lambda_{1} \cup \cdots \cup \Lambda_{p_{1}+1}$ be such that $\Lambda_{k}=\left\{\lambda_{k 1}, \lambda_{k 2}, \ldots, \lambda_{k p_{k}}\right\}, \lambda_{11}=\lambda_{1}$, $\lambda_{k 1} \geq \lambda_{k 2} \geq \ldots \geq \lambda_{k p_{k}}\left(k=1, \ldots, p_{1}+1\right)$, where $p_{1}$ is the number of elements of the list $\Lambda_{1}$ and some of the lists $\Lambda_{i}$ can be empty. Let $\Omega=\Omega_{2} \cup \cdots \cup \Omega_{p_{1}+1}$ with 
$\Omega_{k}=\left\{\omega_{k}, \omega_{k 1}, \omega_{k 2}, \ldots, \omega_{k p_{k}}\right\}, k=2, \ldots, p_{1}+1$. Suppose that the following conditions hold:

i) For each $k=2, \ldots, p_{1}+1$, there exists $0 \leq \mu_{k} \leq \lambda_{1}$ and a symmetric nonnegative matrix $A_{k}$ with spectrum $\Gamma_{k}=\left\{\mu_{k}, \lambda_{k 1}, \ldots, \lambda_{k p_{k}}\right\}$ and diagonal entries $\omega_{k}, \omega_{k 1}, \ldots, \omega_{k p_{k}}$

ii) there exists a $p_{1} \times p_{1}$ symmetric nonnegative matrix $B$ with spectrum $\Lambda_{1}$ and diagonal entries $\mu_{2}, \ldots, \mu_{p_{1}+1}$.

Then there exists a symmetric nonnegative matrix with spectrum $\Lambda$ and diagonal entries $\omega_{1}, \ldots, \omega_{n}$.

Proof. Let $A=A_{2} \oplus \cdots \oplus A_{p_{1}+1}$. From $\left.i\right) A$ is symmetric nonnegative with spectrum $\Gamma_{2} \cup \cdots \cup \Gamma_{p_{1}+1}$. Let $\left\{\mathbf{x}_{2}, \ldots, \mathbf{x}_{p_{1}+1}\right\}$ be an orthonormal set of eigenvectors of $A$ associated, respectively, with $\mu_{2}, \ldots, \mu_{p_{1}+1}$. Then the $n \times p_{1}$ matrix $X$ with $k$ th column $\mathbf{x}_{k}$ satisfies $A X=X \Omega$ for $\Omega=\operatorname{diag}\left(\mu_{2}, \ldots, \mu_{p_{1}+1}\right)$. Each $\mathbf{x}_{k}$ is chosen as in the proof of Theorem 4.1. Then $X$ is entrywise nonnegative. From ii) $C=B-\Omega$ is symmetric nonnegative, and $\Omega+C$ has spectrum $\Lambda_{1}$ and diagonal entries $\mu_{2}, \ldots, \mu_{p_{1}+1}$. Therefore, from Theorem 1.2, the symmetric nonnegative matrix $A+X C X^{T}$ has spectrum $\Lambda$ and diagonal entries $\omega_{1}, \ldots, \omega_{n}$.

EXAmPLE 4.6. Let $\Lambda=\{5,4,0,-3,-3,-3\}$ partitioned as

$$
\Lambda_{1}=\{5,4,0,-3\}, \quad \Gamma_{2}=\Gamma_{3}=\{3,-3\}, \quad \Gamma_{4}=\Gamma_{5}=\{0\}
$$

The symmetric nonnegative matrix

$$
B=\left[\begin{array}{cccc}
3 & 0 & \sqrt{6} & 0 \\
0 & 3 & 0 & \sqrt{6} \\
\sqrt{6} & 0 & 0 & 2 \\
0 & \sqrt{6} & 2 & 0
\end{array}\right]
$$

has spectrum $\Lambda_{1}$ and diagonal entries $3,3,0,0$. Let $\Omega=\operatorname{diag}(3,3,0,0)$ and

$$
X=\left[\begin{array}{cccc}
\frac{\sqrt{2}}{2} & 0 & 0 & 0 \\
\frac{\sqrt{2}}{2} & 0 & 0 & 0 \\
0 & \frac{\sqrt{2}}{2} & 0 & 0 \\
0 & \frac{\sqrt{2}}{2} & 0 & 0 \\
0 & 0 & 1 & 0 \\
0 & 0 & 0 & 1
\end{array}\right], A_{2}=A_{3}=\left[\begin{array}{ll}
0 & 3 \\
3 & 0
\end{array}\right] \text { and } C=B-\Omega
$$


Then we apply Theorem 1.2 to obtain the symmetric nonnegative matrix

$$
\begin{aligned}
A & =\left[\begin{array}{cccccc}
A_{2} & & & \\
& A_{3} & & \\
& & 0 & \\
& & & 0
\end{array}\right]+X C X^{T} \\
& =\left[\begin{array}{cccccc}
0 & 3 & 0 & 0 & \sqrt{3} & 0 \\
3 & 0 & 0 & 0 & \sqrt{3} & 0 \\
0 & 0 & 0 & 3 & 0 & \sqrt{3} \\
0 & 0 & 3 & 0 & 0 & \sqrt{3} \\
\sqrt{3} & \sqrt{3} & 0 & 0 & 0 & 2 \\
0 & 0 & \sqrt{3} & \sqrt{3} & 2 & 0
\end{array}\right]
\end{aligned}
$$

with spectrum $\Lambda$. Observe that no partition works for the criterion in Theorem 1.4.

EXAMPLE 4.7. We construct, from Theorem 4.5, a symmetric nonnegative matrix $B$ with eigenvalues $8,6,3,3$ and diagonal entries $5,5,5,5$. Consider the partition $\Lambda_{1}=\{8,6,3\}, \Lambda_{2}=\{3\}$ with $\Gamma_{2}=\{7,3\}$. Then

$$
B_{1}=\left[\begin{array}{ccc}
7 & \frac{\sqrt{2}}{2} & \frac{\sqrt{2}}{2} \\
\frac{\sqrt{2}}{2} & 5 & 2 \\
\frac{\sqrt{2}}{2} & 2 & 5
\end{array}\right] \text { and } B_{2}=\left[\begin{array}{cc}
5 & 2 \\
2 & 5
\end{array}\right]
$$

are symmetric nonnegative with spectrum $\Lambda_{1}$ and $\Gamma_{2}$, respectively. Hence, the required symmetric nonnegative matrix $B$ is

$$
\begin{aligned}
B & =\left[\begin{array}{llll}
5 & 2 & 0 & 0 \\
2 & 5 & 0 & 0 \\
0 & 0 & 5 & 0 \\
0 & 0 & 0 & 5
\end{array}\right]+\left[\begin{array}{ccc}
\frac{\sqrt{2}}{2} & 0 & 0 \\
\frac{\sqrt{2}}{2} & 0 & 0 \\
0 & 1 & 0 \\
0 & 0 & 1
\end{array}\right]\left[\begin{array}{ccc}
0 & \frac{\sqrt{2}}{2} & \frac{\sqrt{2}}{2} \\
\frac{\sqrt{2}}{2} & 0 & 2 \\
\frac{\sqrt{2}}{2} & 2 & 0
\end{array}\right]\left[\begin{array}{cccc}
\frac{\sqrt{2}}{2} & \frac{\sqrt{2}}{2} & 0 & 0 \\
0 & 0 & 1 & 0 \\
0 & 0 & 0 & 1
\end{array}\right] \\
& =\left[\begin{array}{llll}
5 & 2 & \frac{1}{2} & \frac{1}{2} \\
2 & 5 & \frac{1}{2} & \frac{1}{2} \\
\frac{1}{2} & \frac{1}{2} & 5 & 2 \\
\frac{1}{2} & \frac{1}{2} & 2 & 5
\end{array}\right] .
\end{aligned}
$$

5. On the complex case. In [20], the authors prove a constructive sufficient condition for the existence of a nonnegative matrix with prescribed complex spectrum $\Lambda=\left\{\lambda_{1}, \ldots, \lambda_{n}\right\}$. Now, as in Sections 3 and 4 , we have the following result.

TheOREM 5.1. Let $\Lambda=\left\{\lambda_{1}, \lambda_{2}, \ldots, \lambda_{n}\right\}$ be a list of complex numbers with $\Lambda=\bar{\Lambda}$, $\lambda_{1} \geq \max _{i}\left|\lambda_{i}\right|, i=2, \ldots, n ; \sum_{i=1}^{n} \lambda_{i} \geq 0$ and let $\Lambda=\Lambda_{1} \cup \cdots \cup \Lambda_{p_{1}+1}$, be such that 
$\Lambda_{k}=\left\{\lambda_{k 1}, \lambda_{k 2}, \ldots, \lambda_{k p_{k}}\right\}, \lambda_{11}=\lambda_{1},\left(k=1, \ldots, p_{1}+1\right)$, where $\Lambda_{1}$ is realizable, $p_{1}$ is the number of elements of the list $\Lambda_{1}$ and some lists $\Lambda_{k}$ can be empty. Let $\omega_{2}, \ldots, \omega_{p_{1}+1}$ be real numbers satisfying $0 \leq \omega_{k} \leq \lambda_{1}, k=2, \ldots, p_{1}+1$. Suppose that the following conditions hold:

i) For each $k=2, \ldots, p_{1}+1$, there exists a nonnegative matrix $A_{k}$ with spectrum $\Gamma_{k}=\left\{\omega_{k}, \lambda_{k 1}, \ldots, \lambda_{k p_{k}}\right\}$;

ii) there exists a $p_{1} \times p_{1}$ nonnegative matrix $B$ with spectrum $\Lambda_{1}$ and diagonal entries $\omega_{2}, \ldots, \omega_{p_{1}+1}$.

Then $\Lambda$ is realizable.

Proof. The proof is similar to the proof of Theorem 3.1.

Although the partitions in both results, Theorem 5.1 and Theorem 2.1 in [20], seem to be different, it is easy to see that both Theorems are equivalent. Consider the following example:

EXAmPLE 5.2. Let $\Lambda=\{10,-1+2 i,-1-2 i, 2+3 i, 2-3 i, 3+3 i, 3-3 i\}$. We take the partition $\Lambda=\Lambda_{1} \cup \Lambda_{2} \cup \Lambda_{3}$, where

$$
\Lambda_{1}=\{10,3+3 i, 3-3 i\}, \Lambda_{2}=\{2+3 i, 2-3 i\}, \Lambda_{3}=\{-1+2 i,-1-2 i\},
$$

with

$$
\Gamma_{2}=\{8,2+3 i, 2-3 i\}, \Gamma_{3}=\{4,-1+2 i,-1-2 i\}, \Gamma_{4}=\{4\}
$$

realizable by nonnegative matrices

$$
A_{2}=\left[\begin{array}{lll}
5 & 0 & 3 \\
6 & 2 & 0 \\
0 & 3 & 5
\end{array}\right], \quad A_{3}=\left[\begin{array}{lll}
1 & 1 & 2 \\
4 & 0 & 0 \\
0 & 3 & 1
\end{array}\right], \quad A_{4}=[4]
$$

respectively. We also compute from Theorem 2.1 , the matrix

$$
B=\left[\begin{array}{ccc}
8 & 0 & 2 \\
\frac{17}{3} & 4 & \frac{1}{3} \\
0 & 6 & 4
\end{array}\right]
$$

with spectrum $\Lambda_{1}$ and diagonal entries 8, 4, 4 (corresponding to the Perron eigenvalues of $A_{2}, A_{3}$ and $A_{4}$ ). Let

$$
M=\left[\begin{array}{lll}
A_{2} & & \\
& A_{3} & \\
& & 4
\end{array}\right], \quad X^{T}=\left[\begin{array}{lllllll}
1 & 1 & 1 & 0 & 0 & 0 & 0 \\
0 & 0 & 0 & 1 & 1 & 1 & 0 \\
0 & 0 & 0 & 0 & 0 & 0 & 1
\end{array}\right]
$$


and $C$ be the matrix obtained from $B$ as

$$
C=\left[\begin{array}{ccccccc}
0 & 0 & 0 & 0 & 0 & 0 & 2 \\
\frac{17}{3} & 0 & 0 & 0 & 0 & 0 & \frac{1}{3} \\
0 & 0 & 0 & 6 & 0 & 0 & 0
\end{array}\right]
$$

Then,

$$
A=M+X C=\left[\begin{array}{ccccccc}
5 & 0 & 3 & 0 & 0 & 0 & 2 \\
6 & 2 & 0 & 0 & 0 & 0 & 2 \\
0 & 3 & 5 & 0 & 0 & 0 & 2 \\
\frac{17}{3} & 0 & 0 & 1 & 1 & 2 & \frac{1}{3} \\
\frac{17}{3} & 0 & 0 & 4 & 0 & 0 & \frac{1}{3} \\
\frac{17}{3} & 0 & 0 & 0 & 3 & 1 & \frac{1}{3} \\
0 & 0 & 0 & 6 & 0 & 0 & 4
\end{array}\right] \in \mathcal{C S}_{10}
$$

has the spectrum $\Lambda$.

Acknowledgment. We sincerely thank an anonymous referee for his/her careful reading of the first version and helpful comments.

\section{REFERENCES}

[1] G. Bharali and O. Holtz. Functions preserving nonnegativity of matrices. SIAM J. Matrix Anal. Appl., 30:84-101, 2008.

[2] A. Borobia, J. Moro, and R.L. Soto. Negativity compensation in the nonnegative inverse eigenvalue problem. Linear Algebra Appl., 393:73-89, 2004.

[3] A. Borobia, J. Moro, and R.L. Soto. A unified view on compensation criteria in the real nonnegative inverse eigenvalue problem. Linear Algebra Appl., 428:2574-2584, 2008.

[4] A. Brauer. Limits for the characteristic roots of a matrix IV: Applications to stochastic matrices. Duke Math. J., 19:75-91, 1952.

[5] M. Fiedler. Eigenvalues of nonnegative symmetric matrices. Linear Algebra Appl., 9:119-142, 1974.

[6] A. Horn. Doubly stochastic matrices and the diagonal of a rotation matrix. Amer. J. Math., 76:620-630, 1954.

[7] T. Laffey and E. Meehan. A refinament of an inequality of Johnson, Loewy and London on nonnegative matrices and some applications. Electron. J. Linear Algebra, 3:119-128, 1998.

[8] T.J. Laffey and H. Šmigoc. Construction of nonnegative symmetric matrices with given spectrum. Linear Algebra Appl., 421:97-109, 2007.

[9] R. Loewy and D. London. A note on an inverse problem for nonnegative matrices. Linear Multilinear Algebra, 6:83-90, 1978.

[10] C. Marijuán, M. Pisonero, and R.L. Soto. A map of sufficient conditions for the real nonnegative inverse eigenvalue problem. Linear Algebra Appl., 426:690-705, 2007.

[11] M.E. Meehan. Some results on matrix spectra, Ph.D. thesis. National University of Ireland, Dublin, 1998.

[12] H. Perfect. Methods of constructing certain stochastic matrices II. Duke Math. J. , 22:305-311, 1955. 
[13] N. Radwan. An inverse eigenvalue problem for symmetric and normal matrices. Linear Algebra Appl., 248:101-109, 1996.

[14] O. Rojo and R.L. Soto. Existence and construction of nonnegative matrices with complex spectrum. Linear Algebra Appl., 368:53-69, 2003.

[15] H. Šmigoc. The inverse eigenvalue problem for nonnegative matrices. Linear Algebra Appl., 368:53-69, 2003.

[16] R.L. Soto. Existence and construction of nonnegative matrices with prescribed spectrum. Linear Algebra Appl., 369:169-184, 2003.

[17] R.L. Soto. Realizability criterion for the symmetric nonnegative inverse eigenvalue problem. Linear Algebra Appl., 416:783-794, 2006.

[18] R.L. Soto and O. Rojo. Applications of a Brauer theorem in the nonnegative inverse eigenvalue problem. Linear Algebra Appl., 416:844-856, 2006.

[19] R.L. Soto, O. Rojo, J. Moro, and A. Borobia. Symmetric nonnegative realization of spectra. Electron. J. Linear Algebra, 16:1-18, 2007.

[20] R.L. Soto, M. Salas, and C. Manzaneda. Nonnegative realization of complex spectra. Electron. J. Linear Algebra, 20:595-609, 2010.

[21] G. Soules. Constructing symmetic nonnegative matrices. Linear Multilinear Algebra, 13:241$251,1983$.

[22] J. Torre-Mayo, M.R. Abril-Raymundo, E. Alarcia-Estévez, C. Marijuán, and M. Pisonero. The nonnegative inverse eigenvalue problem from the coefficients of the characteristic polynomial. EBL digraphs. Linear Algebra Appl., 426:729-773, 2007. 